\title{
IoT Based Smart Lighting System using PIR Sensors, Arduino Uno and Thingspeakcloud + Chat Bot and Dashboard to Monitor Home Remotely
}

\author{
Pinak Desai, Nilesh Modi
}

\begin{abstract}
As described in paper [1], I have mentioned solution to smart lighting and security using arduinouno microprocessor, Wi-Fi module and PIR Sensor.In this era, we all are fully dependent on electricity which is non-renewable resource. We must require some solution to save electricity and use electricity wisely. Lighting system is one of the major key component of smart cities or developing cities. Even we need to constantly monitor electricity usage of our home remotely when we are not available at our home. With the development of new technologies like IOT, sensors etc., automation of electricity is easy and it plays very important role in saving electricity. The purpose of this project is to save and monitor your home lights using IOT and other technologies. Here we propose IOT and cloud based smart lighting system and bot+dashboard to monitor you home electricity very efficiently. When the person/persons in your home enters particular area, the load will be activated and when they leave particular area load will be deactivated of that area and this data is also sent to cloud to monitor your home.
\end{abstract}

Keywords:Smart Lighting Solutions, Thingspeak Cloud, PIR Sensor, Chat Bot, Smart Home Dashboard, Arduino Uno

\section{INTRODUCTION}

In this paper, we have mentioned smart lighting solutions with PIR sensors, Arduino Uno, Thingspeak cloud etc.ThingSpeak is Open Source IOT application which helps developers to store and retrieve data from things using HTTP protocol. This system regularly monitors PIR sensors response in rooms using clusters of PIR sensors and response accordingly.Electricity is very important and to make effective use of electricity one need proper solution for lighting control to save electricity.PIR (Passive infrared sensor) sensors actually used to sense availably of any person in the room. It uses infrared signals to sense the person's movement. In this paper I have also mentioned dashboard which helps you in monitoring your smart home lighting system from anywhere in world and it also helps you to effectively manage electricity of all your rooms. Chat Bot is a bot which automatically answers all your questions about smart lighting system of your home. You can talk with your home using chat bot. In this paper, I have mentioned below topics.

Revised Manuscript Received on July 22, 2019

Pinak Desai, Kadi University, Near ITI, Road Number 5, Sector 15, Gandhinagar, Gujarat, India

Dr. Nilesh Modi, Dr. BabasahebAmbedkar Open University, JyotirmayParisar, Opp. Shri Balaji Temple, Sarkhej-Gandhinagar Highway, Chharodi, Ahmedabad, Gujarat, India
1) Introduction

2) Smart Home Lighting System

3) Problems with Current System

4) Thingspeak Cloud

5) Development of ChatBot and Dashboard for Smart Lighting System

6) Communication between C\# ChatBot and Arduino Code using ThingSpeak Cloud

7) Conclusions

8) Future Extension

\section{SMART HOME LIGHTING SYSTEM}

In my previous paper [1], As Shown in figure 1, We have developed Smart Lighting and security solution which uses buzzer, arduinouno microprocessor, PIR sensors, relay and arduino IDE (to write code for arduinouno microprocessor).

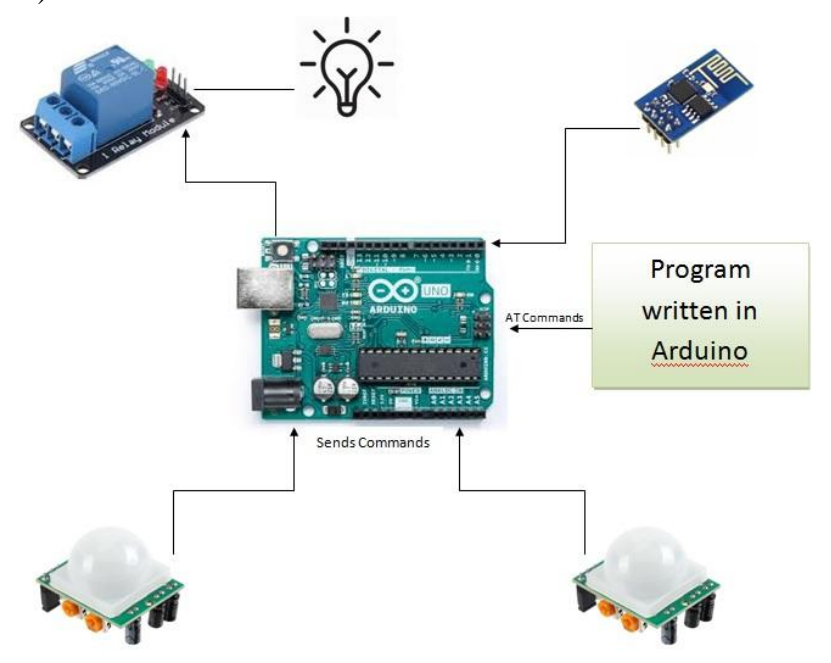

Fig. 1.Smart Lighting System Architecture

As Shown in figure 1, I have used arduinouno, relay, ESP $8266 \mathrm{Wi}-\mathrm{Fi}$ module, PIR sensors etc. As shown in figure when PIR sensor detects any motion, using AT commands arduinouno instructs relay that PIR detected some motion and in action relay will turn on lights.

If your room size is so big and you have connected all lights with same PIR sensor than when someone enters room then all light will turn on. Paper [2] solves this issues by using case studies. But Ideal situation is that when person enters the room not all lights but only lights near to that person must be turn on.

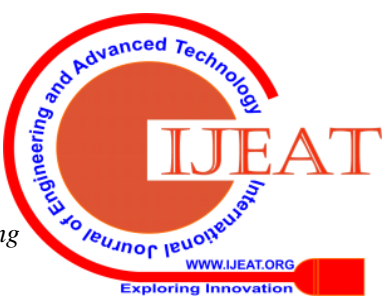




\section{PROBLEMS WITH CURRENT SYSTEM}

With the smart lighting system shown above, you must have some proper solution that helps you in managing your system anywhere in world. Problems with current system are as below:

1) When you are not there in your home and if someone enters the home then alert must be generated in form of message or email that someone entered your room.

2) You must need some numbers like light status of last 30 days or 60 days to analyze usage of your electricity.

3) You must need some solution to access your lights remotely or to get status remotely.

4) You need some visuals like bar chart or split charts to monitor or save electricity of your home.

This paper helps you in solving all problems mentioned above with your current Smart Lighting System.

\section{THINGSPEAK CLOUD}

There are lots of challenges to IOT developers like,

1) How I implement my algorithm on smart devices?

2) How do I collect enough data to build my algorithm?

3) How do I develop my algorithms?

4) How do I deploy my algorithms to the cloud?

The solution of all challenges are Thingspeak cloud. Thingspeak helps IOT developers in analyzing data and perform any operation on data from things. Thingspeak has inbuilt MATLAB for IOT analytics. Things speaks analyze IOT algorithms developed by developers and communicate with things or smart devices. Architecture of thingspeak cloud is shown in figure 2 .

Thingspeak includes rest API or web service that helps you in collecting and storing sensor data in cloud and lets you develop Internet of Things applications. The strength of thingspeak compare to other clouds is that it uses public channel. Another strength of thingspeak is that it is specially designed for IOT developers and it uses Phusion Passenger Enterprise and provides support for python, node.js and ruby.Thingspeak channel allows you to store 8 fields, 4 dedicated field for storing longitude, latitude, description and elevation.

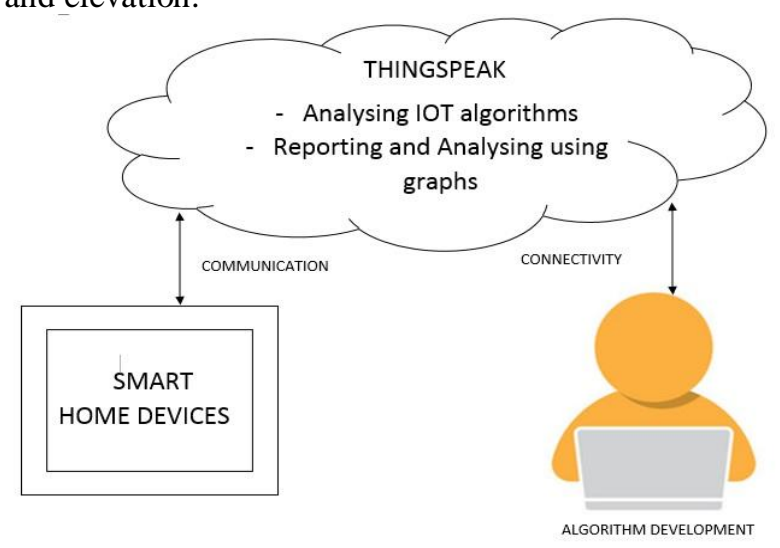

Fig. 2.Thingspeak Cloud Architecture

\section{DEVELOPMENT OF CHATBOT \& DASHBOARD FOR SMART LIGHTING SYSTEM}

This module helps you if you are not available at home and you want to communicate with your home. We have developed this module in ASP.NET C\#. This paper also represents Customized dashboard which let you visualized and analyze your home electricity usage as shown in figure 3 and 4 .

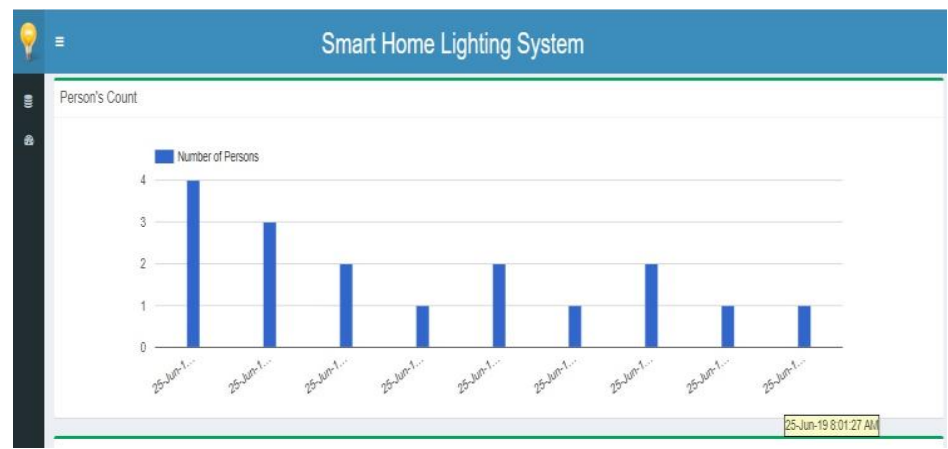

Fig. 3. Bar chart showing Number of Persons in room at specific Time

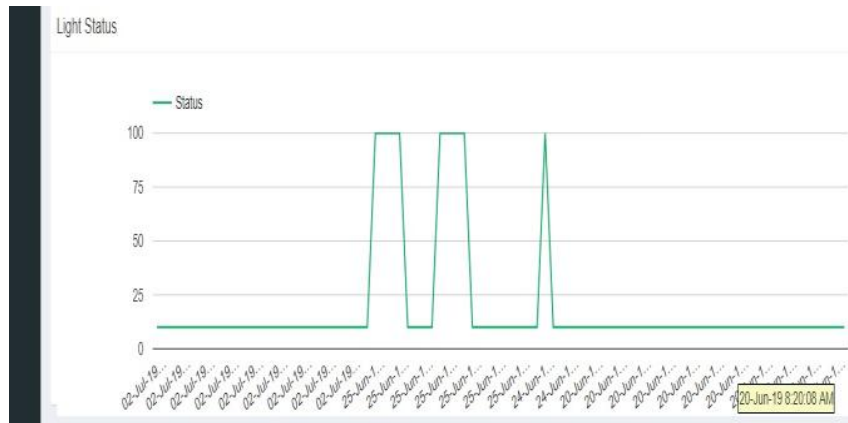

Fig. 4. Line chart showing Light Status of room at specific Time

Figure 3 shows number of persons in room at specific time. As shown in figure there are 1 person in room at 25-Jun-2019 8:01:27 AM.....

Figure 4 shows line chart of light status of my room at specific time. For example at shown in figure 4 the light status of my room at 20-Jun-2019 8:20:08 AM is off.

Chat bot helps you to simulate conversion or chat with your home in natural language. We have also developed chat bot of Smart Lighting System in ASP.NET C\#. Example of Chat bot we have developed in C\# is shown in Figure 6. You can even remotely access you light from chat bot. The whole view of Chat bot is shown in figure 5 .

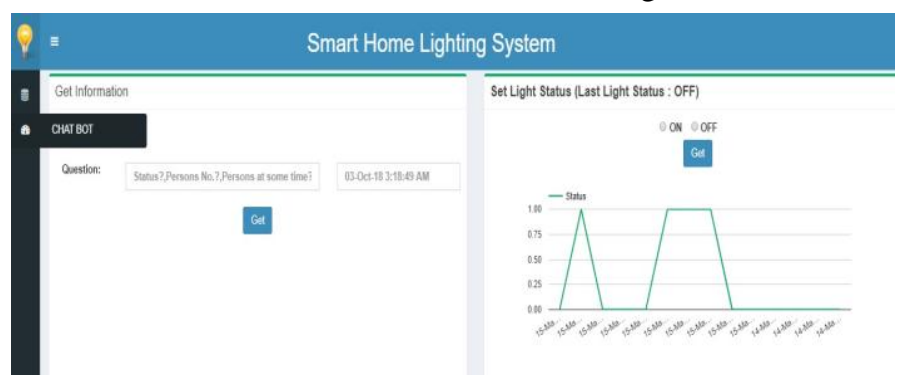

Fig. 5. Chat Bot

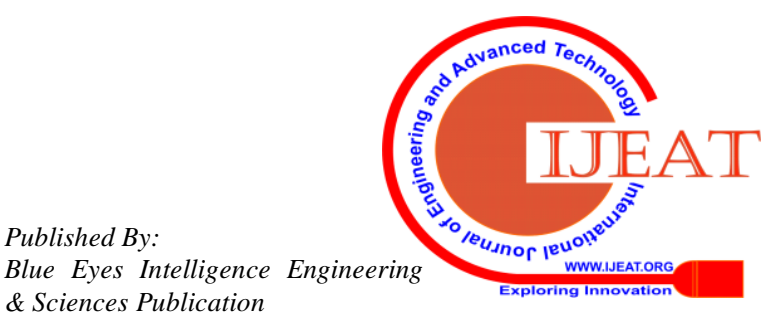


The figure mentioned above shows chat bot view. In first part of chat bot you can communicate and ask questions to your home and chat bot will reply your answers. Example of questions you can ask to Chat Bot are as below,

1) Can I know current light status of my room?

2) Can you tell me how many number of persons are there in my room?

3) Let me know light status of my room on 20-Jun-2019 10:25:08 AM.

4) Please tell me how many persons were there in my room at 01-Jun-2019 5:25:08 AM.

\section{Get Information}

Question:

$$
\begin{array}{ll}
\text { How many Person was there in my room at } \quad \text { 25-Jun-19 8:54:40 AM }
\end{array}
$$

\section{Get}

\section{Number of Persons in the room at 25-Jun-19 8:54:40 AM are : 4}

Fig. 6. Chat Bot Questions and Answers

As you can see in figure 6, I have ask my home a question that how many persons are there in my room at 25-Jun2019 8:54:40 AM and my home answers that number of persons in your room at 25-Jun-2019 8:54L40 AM are 4.

You can even set light status of your room even when you are not available at home. Figure 7 shows that we can on or off lights of our room. Line chart below light status shows real time picture of your room. When your turn on your light than line of this graph will reflect to on.

Set Light Status (Last Light Status : OFF)

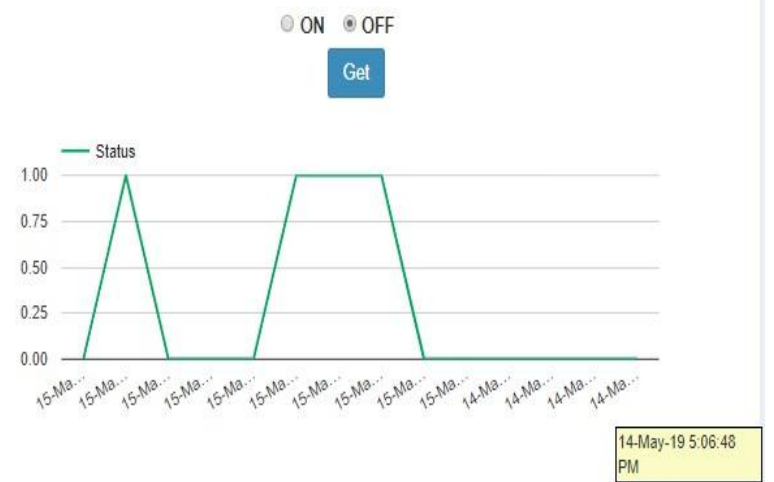

Fig. 7. Setting Light Status to Off

\section{COMMUNICATION BETWEEN C\# CHAT BOT AND ARDUDINO CODE USING THINGSPEAK CLOUD}

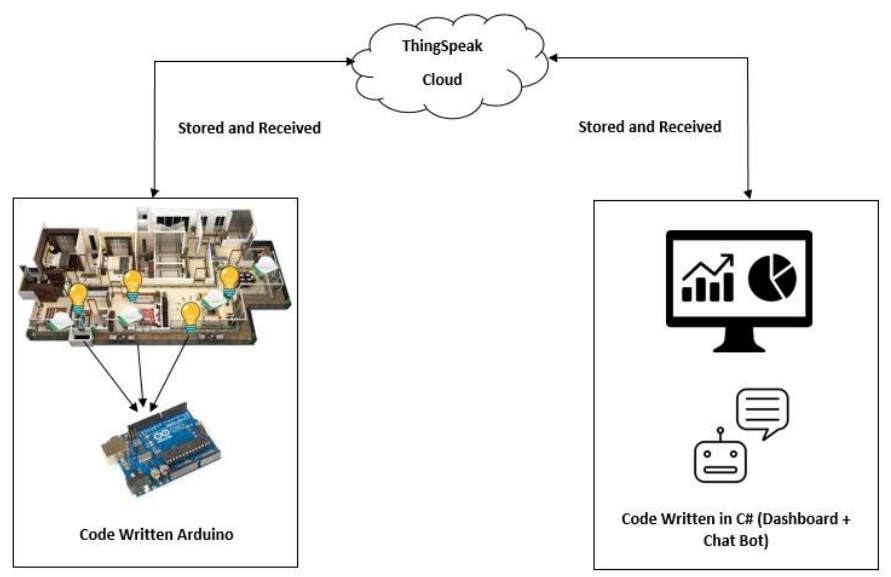

Fig. 8. Communication between chat bot and thingspeak

As shown in figure 8 thingspeak plays important role in communication between c\# code and arduino code.

Arduino code when any events occur updates thingspeak field by using update request of thingspeak continuously in loop. So when C\# code want to communicate with arduino it concurrently sends get or update request to thingspeak cloud.

For example if we ask question to chat bot that can you tell me how many number of persons are there in my room?, then c\# code will send get request like http://api.thingspeak.com/channels/xxxxxx/field/3/last.html to thingspeak cloud and thingspeak will return last value of field 3 to c\# then c\# will display this value to the user.

The code shown below shows how get request is sent to thingspeak cloud using C\#. We have downloaded json data from thingspeakapi and converted JSON to datatable which helps us in performing operation in $\mathrm{C \#} \mathrm{like} \mathrm{setting} \mathrm{value} \mathrm{to}$ label, getting last value from thingspeak and displaying that value to label etc. and as shown below I have converted GMT to IST

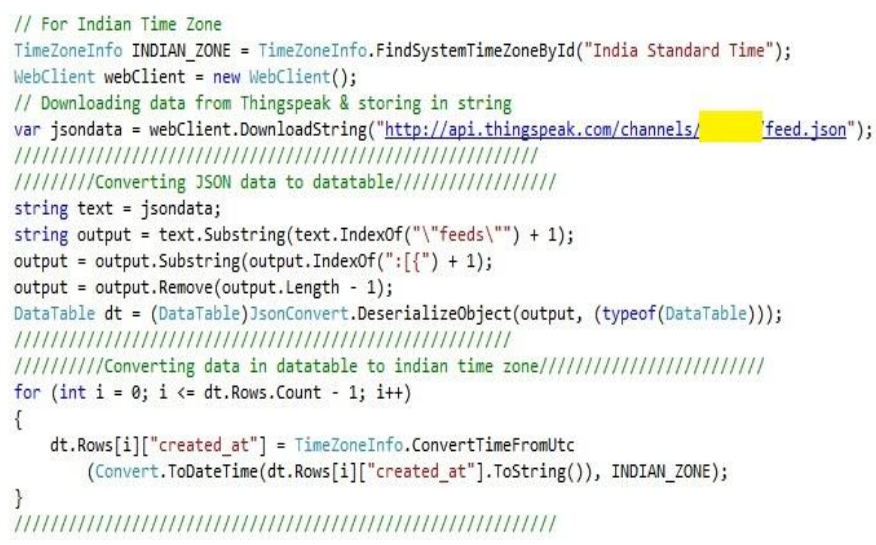

Same way we can use update request that help us in updating data of field in thingspeak cloud as shown below, 

to Monitor Home Remotely

//////////////////Code for updating light status

string apiKey;

double tagValue;

string server = "https://api, thingspeak. com/";

string webMethod;

string uri;

var webclient $=$ new Webclient();

apikey = $\quad$;//Write Api key of your channel

////Updating Field 3 of thingspeak cloud//////////////

webMethod = "update?api_key=" + apiKey + "\&field3="+rblPerfIndex.SelectedValuet" ; uri $=$ server + weblethod;

webclient.UploadString(uri, "POST", "");

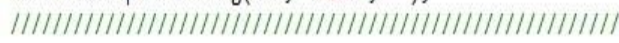

\section{CONCLUSION}

As described in this paper, this paper is extension of Smart Lighting System. We have developed chat bot and dashboard that helps in to communicate with your home. Thingspeak is a key feature of any IOT application that helps us in analyzing out things data with inbuilt MATLAB function that help us with generating reports and graphs. You can also access lights of your home from anywhere in this world. We have used PIR sensors, Arduino Uno, relay for developing smart lighting system.

\section{FUTURE EXTENSION}

We are planning to send SMS or alert by email when any event occurs like light status or someone enters the room. Brightness of the light of the room must be according to weather. When weather is bright that light must be set to low brightness and when whether is cloudy then brightness of light must be high. To increase the range of PIR sensors we can use amplifier gain adjusted according to the application. The achievable range is however mostly limited by sensor noise, the usual means to achieve a wide sensor range is a different (single zone, large aperture) Fresnel lens.The PIR sensors normally have parabolic mirrors or Fresnel lenses that have essentially an infinite focus (which turns out to be about 30 - 50 feet.). You will need to either get a PIR with different focal length or experiment with the threshold level of the amplifier within the unit. Note that they usually have some type of sample-and-hold amp or other filter as well to prolong the response. Also, there are often multiple little sensors with multiple mirrors on a wide-angle sensor. They are usually wired so activation of one trips the overall sensor. Also, those multiple sensors could be wired as difference amps so temp change doesn't cause a signal.

\section{REFERENCES}

1. http://ijrar.com/upload_issue/ijrar_issue_2024.pdf

2. "Smart Lighting Solution using Clusters of Sensors (Problems with Limited Sensors) and Analyzing Networks for Smart Lighting Solution”,Pinak Desai, Nilesh Modi,Volume-8 Issue-6S, April 2019

3. "Automated street lighting system using IoT",PrashanthKeni, Shaik Mohammed Wajid, Syed Zuber Ahmad, Rahimunnisa and ShruthiK,IJARIT, Volume 4,Issue 3

4. "Density Based Smart Lighting System Using IoT",N.S.Yoganathan , G.LakshmiPriya , R.Monisha, A.Lavanya and S.Kowsalya,IJAREEIE, Volume 7, Issue 2, February-2018

5. "Implementation of Home Automation with Thingspeak Cloud",DalliSai Suresh, SivahAkash, International Journal of Scientific \& Engineering Research Volume 9, Issue 9, September-2018
6. "Internet of Things (IoT) based Sensors to Cloud system using ESP8266 and Arduino Due", Mr. NerellaOme and Mr. G. Someswara Rao, IJARCCE, Voume 5, Issue 10, October-2016

7. "Smart Home Energy Management System Including Renewable Energy Based on ZigBee and PLC", Jinsoo Han, Chang-Sic Choi, Wan-Ki Park, Ilwoo Lee, and Sang-Ha Kim, IEEE Transactions on Consumer Electronics, Vol. 60, No. 2, May 2014 Contributed Paper

8. "Design and Implementation of User Interactive Wireless Smart Home Energy Management System", AryadeviRemanideviDevidas, Subeesh T. S, ManeeshaVinodiniRamesh,Center for Wireless Networks and Applications Amrita VishwaVidyapeethamAmritapuri, Kollam, India

9. https://indico.cern.ch/event/654636/contributions/2834242/attachment s/1636045/2610243/MATLAB_IoT_public.pdf

\section{AUTHORS' PROFILES}

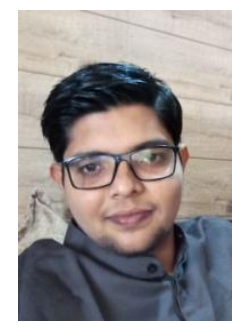

Pinak N. Desaiborned at Ahmedabad (Gujarat), India, 3r d March 1991. After graduating in computer applications (BCA) from Kadi University, Gandhinagar (Gujarat), India in 2011, he took his master degree in computer applications, (MCA) from Gujarat Technological University, Gandhinagar (Gujarat), India in 2013. Recently he is doing P.H.D in the area of - Smart Home from KSV University, Gandhinagar(Gujarat), India since 2016.He is senior programmer in National Informatics Centre (NIC), Gandhinagar (Gujarat), India. Before NIC, he was working in Gujarat Informatics Ltd. As a programmer trainee and handling government projects.

He is Microsoft Technology Associate (MTA) (Software Development Fundamentals).His current area of research are Smart Home, Smart Grid, and Energy Management Systems.

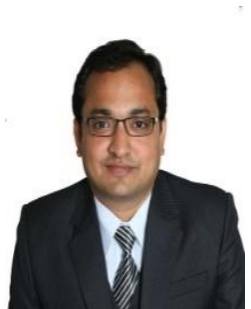

Dr NileshModi, having rich experience of around 12 years in academics and IT industry. He is holding Doctorate in E-Security (Computer Science and Application).Continuing his research on Cyber Security, presently he is pursuing post-doctoral research on Wireless Communication and Security and certification as an Ethical Hacking.He is working as a recognized research supervisors for $\mathrm{Ph} . \mathrm{D}$ and M.Phil. Programme for different universities in India. He has reviewed number of Ph.D., M.Phil and M.Tech thesis from different universities in India and abroad also evaluated the scholars for the same.He is also serving as a manuscript reviewer and program committee member for different research journals and conferences of national and international repute in the world

He has very good number of research under his name and presented more than 75 research papers in International and National Journals and Conferences. He has delivered number of expert talk on eSecurity and hacking in National and International Conferences. Recently He is working in Dr. BabasahebAmbedkar Open University as Computer Science Professor. 